\title{
Follow-up issues in children with mild traumatic brain injuries
}

\author{
Katarzyna Kania, MPH, ${ }^{1}$ Kashif Ajaz Shaikh, MD, ${ }^{1,2}$ Ian Kainoa White, MD,,2 and \\ Laurie L. Ackerman, MD ${ }^{1,2}$ \\ 1'Indiana University School of Medicine; and ${ }^{2}$ Goodman Campbell Brain and Spine, Indiana University Department of \\ Neurological Surgery, Indianapolis, Indiana
}

\begin{abstract}
OBJECTIVE Concerns about mild traumatic brain injury (mTBI) have increased in recent years, and neurosurgical consultation is often requested for patients with radiographic abnormalities or clinical findings suspicious for mTBI. However, to the authors' knowledge, no study has used the Acute Concussion Evaluation (ACE) tool to systematically evaluate the evolution of symptoms in patients with mTBI during neurosurgical follow-up. The goal in this study was to evaluate symptom progression in pediatric patients referred for neurosurgical consultation by using the ACE, as endorsed by the Centers for Disease Control and Prevention.
\end{abstract}

METHODS The authors performed a retrospective review of records of consecutive pediatric patients who had presented to the emergency department, were diagnosed with possible mTBI, and were referred for neurosurgical consultation. Outpatient follow-up for these patients included serial assessment using the ACE. Data collected included the mechanisms of the patients' injuries, symptoms, follow-up duration, and premorbid conditions that might potentially contribute to protracted recovery.

RESULTS Of 91 patients identified with mTBI, 58 met the inclusion criteria, and 33 of these had sufficient follow-up data to be included in the study. Mechanisms of injury included sports injury (15 patients), isolated falls (10), and motor vehicle collisions (8). Ages ranged from 5 to 17 years (mean age 11.6 years), and 29 of the 33 patients were male. Six patients had preinjury developmental and/or psychiatric diagnoses such as attention deficit hyperactivity disorder. Seventeen had negative findings on head CT scans. The first follow-up evaluation occurred at a mean of 30 days after injury. The mean number of symptoms reported on the ACE inventory at first follow-up were 3.2; 12 patients were symptom free. Patients with positive head CT findings required longer follow-up: these patients needed 14.59 weeks, versus 7.87 weeks of follow-up in patients with negative findings on head CT scans $(p<0.05)$.

CONCLUSIONS The data suggest that patients with mTBI, particularly those with developmental and/or psychiatric comorbidities and concurrent cerebral or extracranial injury, often report symptoms for several weeks after their initial injury. Serial ACE assessment permits systematic identification of patients who are experiencing continued symptoms, leading to appropriate patient management and referral.

http://thejns.org/doi/abs/10.3171/2016.1.PEDS15511

KEY WORDS traumatic brain injury; concussion; head injury; patient outcome assessment; acute concussion evaluation; trauma

$\mathrm{M}$ ILD traumatic brain injury (mTBI) has been increasingly recognized as a public health issue as public awareness of contact sport and combat injuries increases. ${ }^{9-11,15}$ It may include diagnoses such as concussion, linear skull fracture, and traumatic subarachnoid hemorrhage. In pediatric patients, mTBI should be given special attention because significant injury can occur with lower energy impact. During the last decade, the rate of emergency department visits for sports- and recreation-related injuries in which a diagnosis of concussion or mTBI was made, alone or in combination with other injuries, rose by $57 \%$ among children and adolescents age

ABBREVIATIONS ACE = Acute Concussion Evaluation; $C D C=$ Centers for Disease Control; Child SCAT3 = Child Sport Concussion Assessment Tool, 3rd edition; ICD = International Classification of Disease; ImPACT = Immediate Postconcussion Assessment and Cognitive Test; $\mathrm{mTBI}=$ mild traumatic brain injury; SCAT3 = Sport Concussion Assessment Tool, 3rd edition.

SUBMITTED August 21, 2015. ACCEPTED January 6, 2016.

INCLUDE WHEN CITING Published online April 8, 2016; DOI: 10.3171/2016.1.PEDS15511. 
19 years or younger. ${ }^{3}$ Falls and blunt trauma caused 55\% and $24 \%$ of TBIs, respectively, in children younger than 15 years. Motor vehicle crashes were the third overall leading cause of TBI, and assaults were responsible for $3 \%$ of TBIs in this age group. ${ }^{3}$

Neurosurgical consults are often requested when there is radiographic evidence of cerebral or calvarial injury, and neurosurgeons are often the providers who follow the patient after the acute hospitalization or emergency room visit. As a result, it is crucial for neurosurgeons to have an understanding of the follow-up outcomes and management of pediatric patients with mTBI to provide appropriate patient recommendations, referrals, and safe return to school as well as return to play in sports, if applicable. Tools such as the Sport Concussion Assessment Tool, 3rd edition (SCAT3), Child Sport Concussion Assessment Tool, 3rd edition (Child SCAT3) and Immediate Postconcussion Assessment and Cognitive Test (ImPACT) have been used to evaluate athletes and guide return to play. However, many children are not seen in the context of sports injuries, or are too young for these tools to be meaningful in guiding the clinician in the context of return to school and playground activities.

The "Heads Up: Brain Injury in Your Practice" toolkit available through the Centers for Disease Control (CDC) website offers a number of resources for dealing with concussion in both sport and nonsport contexts. Among the tools available in the public domain for health care providers are the Acute Concussion Evaluation (ACE) tools. Two versions of this tool are available-one for use in emergency departments and the other for physician/clinician office use. These tools collect information on the following: 1) injury characteristics; 2) a symptoms checklist divided into physical, cognitive, emotional, and sleep disorders problems; and 3) risk factors for protracted recovery such as history of previous concussion, headache history, or presence of developmental or psychiatric comorbidities. To our knowledge, no study has systematically evaluated symptoms using the physician/clinician office use version of the ACE tool during follow-up in patients with mTBI requiring neurosurgical consultation.

The goals of this study were as follows: 1) to evaluate physical, cognitive, emotional, and sleep symptoms in pediatric patients with mTBI by using serial ACE assessments; 2) to determine the impact of developmental and psychiatric history on mTBI recovery; 3 ) to examine the effect of cerebral or calvarial injury on mTBI recovery; and 4) to demonstrate the feasibility and utility of using ACE assessments for symptom comparison over time.

\section{Methods}

The study protocol was approved by the institutional review board at Indiana University School of Medicine. Data were collected as part of a retrospective chart review of children with mTBI. Initially, 91 pediatric patients cared for by the senior author who had presented to the Riley Hospital for Children's Emergency Department in Indianapolis, Indiana, and who were consecutively admitted with International Classification of Disease (ICD) codes for mTBI of 850.0, 850.1, 850.9, and 854 requiring neuro- surgical consultations in 2012 and 2013 were included (Table 1). Patients were identified by the ICD code assigned at the time of admission by the senior author. Criteria for assigning the codes $850.0,850.1$, and 850.9 were the presence of any alteration in consciousness or altered assessment of mentation. The ICD code 854 was also searched to capture any other patients who might have been missed, or who might not have exhibited early symptoms.

Inclusion criteria were 1) age at injury 5 to 18 years; 2) documentation of mTBI diagnosis on presentation to the Riley Hospital for Children's Emergency Department or another hospital emergency department, if transferred; 3) documentation of a neurosurgical consult; and 4) Englishspeaking patient. Exclusion criteria included the absence of an ACE assessment at follow-up. The ACE assessment was performed at each outpatient visit. Retrospective electronic chart review and appropriate statistical analysis were performed. Outpatient follow-up included serial assessment with the ACE Physician/Clinician Office Version available on the CDC website (www.cdc.gov/headsup/pdfs/providers/ ace-a.pdf). Figure 1 shows a copy of the form.

\section{Results}

Ninety-one patients with mTBI in 2012 and 2013 were identified. Thirty-eight patients were less than 5 years of age, and 8 patients lacked available ACE documentation on follow-up. These 46 patients were excluded from the study, leaving 45 patients who met inclusion criteria. An additional 12 patients were lost to follow-up (did not keep any posthospitalization clinic appointments), leaving 33 patients with reliable data for study (Table 2). The mean patient age was 11.6 years (range 5-17 years). Twenty-nine $(87 \%)$ were male. Mechanisms of injury included sports (15 patients), isolated falls (10), and motor vehicle crash (8). Preinjury developmental and/or psychiatric diagnoses (e.g., attention deficit hyperactivity disorder) had been made in 6. Sixteen had positive findings on head CTs (Table 3).

All patients were scheduled to be seen in clinic, and the first follow-up occurred at a mean of 30 days after injury. Eighteen patients required only 1 follow-up visit. The mean number of symptoms reported at first follow-up on ACE inventory was 3.2. The most prevalent symptoms at first follow-up were headaches (58\%), irritability (27\%), dizziness (24\%), feeling mentally foggy (18\%), fatigue $(18 \%)$, sleeping more than usual (18\%), sadness (15\%), and difficulty concentrating (15\%) (Table 4). Symptoms were reported in the physical, cognitive, emotional, and sleep categories by $60 \%, 24 \%, 27 \%$ and $30 \%$ of patients, respectively. Twelve patients were symptom-free at first followup. The average number of follow-up appointments for all

TABLE 1. The ICD codes for $\mathrm{mTBI}$

\begin{tabular}{cl}
\hline ICD Code & \multicolumn{1}{c}{ Description } \\
\hline 850.0 & Concussion w/ no loss of consciousness \\
\hline 850.1 & Concussion w/ loss of consciousness \\
\hline 850.9 & Concussion unspecified \\
\hline 854 & $\begin{array}{c}\text { Intracranial injury of other \& unspecified nature w/o open } \\
\text { intracranial wound }\end{array}$ \\
\hline
\end{tabular}




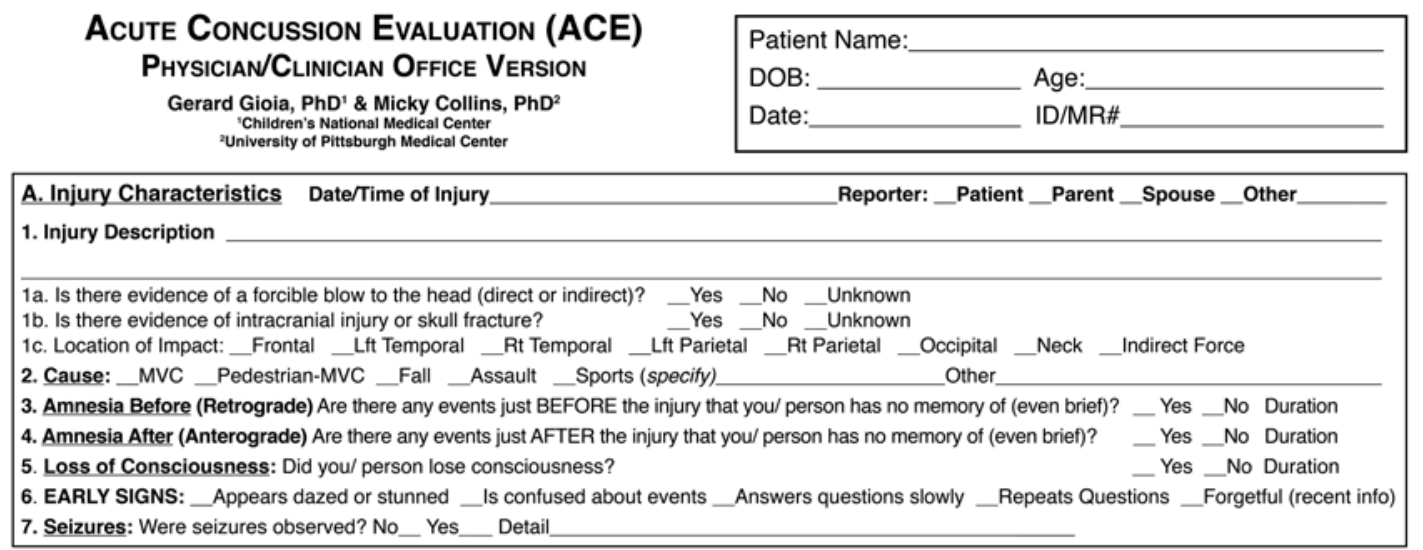

\section{B. Symptom Check List* Since the injury, has the person experienced any of these symptoms any more than usual today or in the past day?} Indicate presence of each symptom $(0=\mathrm{No}, 1=\mathrm{Yes})$.

'Lovell \& Collins, 1998 JHTR

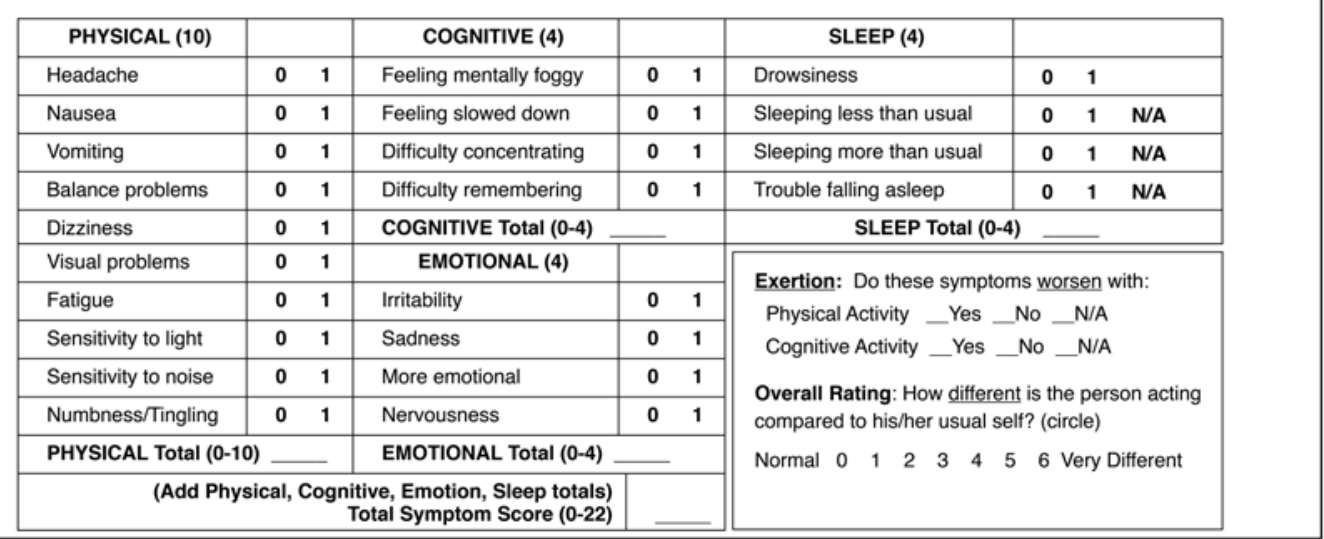

\begin{tabular}{|c|c|c|c|c|c|c|}
\hline \multicolumn{7}{|c|}{ C. Risk Factors for Protracted Recovery (check all that apply) } \\
\hline Concussion History? $\mathrm{Y}_{\ldots} \mathrm{N}_{\mathrm{N}}$ & $\sqrt{ }$ & Headache History? Y__ $\mathrm{N}_{-}$ & $\sqrt{ }$ & Developmental History & $\sqrt{ }$ & Psychiatric History \\
\hline Previous \# $1 \begin{array}{llllll} & 2 & 3 & 4 & 5 & 6+\end{array}$ & & Prior treatment for headache & & Learning disabilities & & Anxiety \\
\hline \multirow{2}{*}{$\begin{array}{l}\text { Longest symptom duration } \\
\text { Days_Weeks__Months__ Years__ }\end{array}$} & & \multirow{3}{*}{$\begin{array}{l}\text { History of migraine headache } \\
\text { - Personal } \\
\text { _ Family }\end{array}$} & & \multirow{2}{*}{$\begin{array}{l}\text { Attention-Deficit/ } \\
\text { Hyperactivity Disorder }\end{array}$} & & Depression \\
\hline & & & & & & Sleep disorder \\
\hline $\begin{array}{l}\text { If multiple concussions, less force } \\
\text { caused reinjury? Yes__ No }\end{array}$ & & & & $\begin{array}{l}\text { Other developmental } \\
\text { disorder }\end{array}$ & & Other psychiatric disorder \\
\hline
\end{tabular}

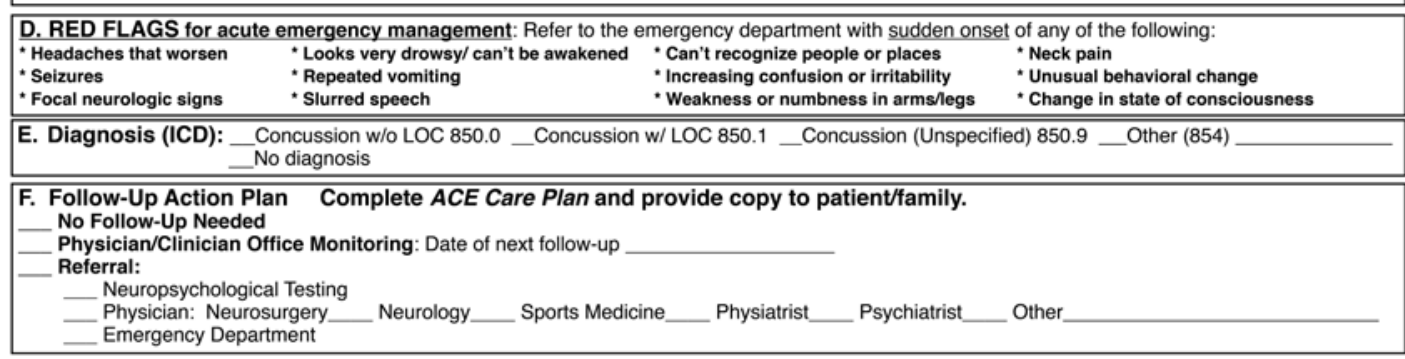

ACE Completed by:

This form is part of the "Heads Up: Brain Injury in Your Practice" tool kit developed by the Centers for Disease Control and Prevention (CDC).

FIG. 1. The ACE tool, Physician/Clinician Office version. Reproduced with permission from the CDC website and Langlois JA. Heads up: facts for physicians about mild traumatic brain injury, 2010 (http://www.cdc.gov/headsup/pdfs/providers/ace-a.pdf [Accessed March 15, 2016]).

patients was 1.67 visits and the average duration of followup was 11.13 weeks.

Patients without developmental and/or psychiatric comorbidities averaged 2.7 symptoms at first follow-up, whereas the 6 patients with developmental and/or psy- chiatric comorbidities averaged 5.7 symptoms $(\mathrm{p}=0.16)$. Patients with developmental and/or psychiatric comorbidities required extended follow-up, requiring 1.83 visits, versus 1.63 visits in patients without these comorbidities ( $p$ $=0.36$ ). These patients with comorbidities also required 
TABLE 2. Admission characteristics of study sample

\begin{tabular}{ll}
\hline \multicolumn{1}{c}{ Characteristic } & Value (\%) \\
\hline Total no. of patients & 33 \\
\hline Mean age in yrs, range & $11.6,5-17$ \\
\hline Sex, male & $29(87)$ \\
\hline Mechanism of injury & $15(45)$ \\
\hline Sports & $10(30)$ \\
\hline Isolated falls & $8(24)$ \\
\hline Motor vehicle crash & $6(18)$ \\
\hline Preinjury psychiatric diagnosis & $16(48)$ \\
\hline Positive head CT*
\end{tabular}

* Evidence of subarachnoid hemorrhage or skull fracture.

11.91 weeks versus 11.13 weeks of follow-up in patients without these comorbidities $(p=0.42)$. These results were not statistically significant. Patients reporting a significant symptom burden persisting longer than 1-2 visits were referred on for additional testing and follow-up visits. Three of the 6 patients with developmental and/or psychiatric comorbidities were referred to other providers, including outpatient rehabilitation for postconcussive symptoms, neurology for ongoing headache management, or sports medicine for return to play. Thus, their length of follow-up may not have been accurately captured because their care was transferred to other physicians.

Patients with negative results on head CT averaged 2.8 symptoms, and patients with skull fracture or intracranial blood averaged 3.6 symptoms at follow-up $(p=0.31)$. Patients with positive head CT results required 1.81 visits, versus 1.53 visits in patients with negative results on head $\mathrm{CT}(\mathrm{p}=0.17)$. These patients with positive CT findings also required 14.59 weeks, versus 7.87 weeks of followup in patients with negative head CT findings $(\mathrm{p}<0.05)$ (Table 5).

TABLE 3. Location and type of finding on admission head CT

\begin{tabular}{cl}
\hline Finding & No. (\%) \\
\hline Total no. of patients & 33 \\
\hline Positive head CT & $16(48)$ \\
\hline Fracture location & $3(19)$ \\
\hline Frontal & $2(13)$ \\
\hline Basilar & $3(19)$ \\
\hline Lt temporal & $2(13)$ \\
\hline Rt temporal & $4(25)$ \\
\hline Lt parietal & $1(6)$ \\
\hline Rt parietal & $2(6)$ \\
\hline Occipital & $1(6)$ \\
\hline Cervical spine & $2(13)$ \\
\hline Facial & $1(6)$ \\
\hline Contusion & $1(6)$ \\
\hline Lt temporal & $4(25)$ \\
\hline Bifrontal &
\end{tabular}

TABLE 4. Frequencies versus type of mTBI symptoms at first follow-up

\begin{tabular}{|c|c|}
\hline Type of Symptom & No. $(\%)$ \\
\hline Total no. of patients & 33 \\
\hline Physical & $20(60)$ \\
\hline Headache & $19(58)$ \\
\hline Nausea & $1(3)$ \\
\hline Vomiting & 0 \\
\hline Balance problem & $5(15)$ \\
\hline Dizziness & $8(24)$ \\
\hline Visual problems & $4(12)$ \\
\hline Fatigue & $6(18)$ \\
\hline Sensitivity to light & $2(6)$ \\
\hline Sensitivity to noise & $3(9)$ \\
\hline Numbness/tingling & 0 \\
\hline Cognitive & $8(24)$ \\
\hline Feeling mentally foggy & $6(18)$ \\
\hline Feeling slowed down & $4(12)$ \\
\hline Difficulty concentrating & $5(15)$ \\
\hline Difficulty remembering & $4(12)$ \\
\hline Emotional & $9(27)$ \\
\hline Irritability & $9(27)$ \\
\hline Sadness & $5(15)$ \\
\hline More emotional & $4(12)$ \\
\hline Nervousness & $4(12)$ \\
\hline Sleep & $10(30)$ \\
\hline Drowsiness & $1(3)$ \\
\hline Sleeping less than usual & $4(12)$ \\
\hline Sleeping more than usual & $6(18)$ \\
\hline Trouble falling asleep & $4(12)$ \\
\hline
\end{tabular}

\section{Discussion}

The 4th International Conference on Concussion in Sport was held in Zurich in November 2012. At that conference, a concussion was defined as "a complex pathophysiological process affecting the brain, induced by biomechanical forces." ${ }^{12}$ Resolution of the clinical and cognitive symptoms of concussion typically follows a sequential course, which may be prolonged in some cases. ${ }^{6,12,15}$ Postconcussive syndrome or persistent concussive symptoms classically consist of sequelae that may occur, resulting in somatic, cognitive, and neuropsychological issues. These postconcussive-like symptoms are also seen in other types of mTBI, such as linear skull fractures, in which the patient may also have symptoms concordant with those described as concussion. The incidence of these symptoms in children ranges from $6 \%$ to $25 \%$, but they usually improve within 1 week. ${ }^{16}$ However, symptoms may persist for weeks or months in some patients, with as many as $58.5 \%$ of individuals symptomatic at 1 month, $11 \%$ at 3 months, and $2.3 \%$ beyond 1 year, according to a study published in Pediatrics. ${ }^{1}$ Studies like these serve to highlight the importance of patient follow-up and the identification of patients struggling with protracted recovery. 
TABLE 5. The mean number of symptoms and duration of follow-up by psychiatric comorbidities and head CT results

\begin{tabular}{lrcccc}
\hline & & $\begin{array}{c}\text { Mean No. of } \\
\text { Cymptoms at }\end{array}$ & \multicolumn{2}{c}{$\begin{array}{c}\text { Mean Duration of } \\
\text { Collow-Up }\end{array}$} \\
\cline { 5 - 6 } \cline { 4 - 5 } Psychiatric comorbidities & 6 & 5.7 & 1.8 & 11.91 \\
\hline No comorbidities & 27 & 2.7 & 1.6 & 11.13 \\
\hline Head CT w/ fracture & 16 & 3.6 & 1.8 & $14.59^{*}$ \\
\hline Normal head CT findings & 17 & 2.8 & 1.5 & $7.87^{*}$ \\
\hline
\end{tabular}

Appt $=$ appointment.

${ }^{*} p<0.05$.

Our findings suggest that patients with radiographic abnormalities on initial imaging, as well as those with preexisting developmental and/or psychiatric comorbidities, trended toward a protracted recovery following mTBI, as evidenced by more symptoms on administration of the ACE inventory and a statistically significant extended length of follow-up in the patients with positive CT findings. All patients with developmental and/or psychiatric comorbidities had attention deficit hyperactivity disorder, and 2 patients had concurrent depression and bipolar disorder diagnoses, respectively. Although only duration of follow-up in patients with positive versus negative CT findings achieved statistical significance, there was a distinct trend for those patients with developmental and/or psychiatric comorbidities to report more symptoms than their counterparts without these comorbidities.

A great deal of attention has been directed to the evaluation of patients with mTBI when "return to play" is an issue. However, children who are not involved in team sports also suffer mTBI injuries. Experts at the 4th International Conference on Concussion in Sport in Zurich recommended that children with concussion should be managed conservatively, with an emphasis on return to school before return to play. ${ }^{12}$ Although there are established criteria and resources regarding return to play for athletes, there is limited guidance and lack of a structured systematic return to school protocol for nonathletes and school-age children. Guidelines suggest cognitive rest and academic accommodations, such as reduced workload and extended time for tests. ${ }^{7}$ However, serial systematic evaluation of the patient is required to understand the level of symptom burden and to translate symptomatology to appropriate treatment and management recommendations. Moreover, the newest tests, the SCAT3 and Child SCAT3, remain more applicable to sports injuries, limiting their utility in nonsports injuries and younger children.13,14 The ACE tool endorsed by the CDC evaluates children in a nonsports-injury context..$^{2,4,5}$ The clinician's office version systematically guides the physician through an assessment of injury characteristics, a symptoms checklist, and risk factors for protracted recovery. This allows for a structured evaluation resulting in early identification of children who are having difficulty with recovery, while educating the parents and provider on symptoms to watch for in the recovery period. This in turn allows for early referral to appropriate providers (e.g., outpatient rehabilitation programs, neuropsychological testing) and provides evi- dence to support academic accommodations and/or interventions in school-age children with protracted recoveries.

\section{Serial ACE}

The ACE tool may be used for systematic serial evaluations of pediatric patients with mTBI. The form was developed to enable physicians to use an evidence-based protocol as an initial and serial evaluation of patients suspected to have mTBI. It includes 3 major components for evaluation: characteristics of injury, type and severity of symptoms, and risk factors for protracted recovery. The symptoms checklist is divided into 4 domains: physical, cognitive, emotional, and sleep (Fig. 1)., ${ }^{2,45}$ It queries whether the symptoms worsen with physical or cognitive activity. We have found it useful to ask both the patient and the parent or caregiver items from the symptom inventory, because sometimes the patient may minimize symptoms that the parent notes, and/or vice versa. Risk factors for protracted recovery include number and characteristics of previous concussions; history of previous headaches or migraines; developmental history, including assessment of learning disabilities and attention deficit hyperactivity disorder; as well as psychiatric comorbidities such as a history of anxiety, depression, sleep disorder, or other psychiatric condition.

In this study, the ACE was administered by the senior author during the outpatient clinic visit(s). It took approximately 2 minutes to ask the patient and family the questions on the form, which were concentrated on the symptom checklist in section B (Fig. 1). The injury characteristics in section A had been previously gathered as part of the admission process during the initial hospitalization, as had the risk factors for protracted recovery in section C.

Serial assessments of the frequency and duration of symptoms guide the clinician in providing care and appropriate referrals. Persistent reports of ongoing symptomatology as well as knowledge of risk factors for protracted recovery provide a basis for early identification of at-risk patients, enabling more timely referrals for physical and cognitive rehabilitation, neuropsychological testing, and implementation of school accommodations. The tool is available in the public domain through the CDC website, takes minutes to administer, and does not add to the cost of patient care. Moreover, the assessment is concrete and reproducible, focusing the clinician on important historical elements and allowing both the clinician and parents to gauge the quality and frequency of symptoms progressively over time.

The ACE care plan serves to educate parents on gradual return to daily activities, school, and sports, while allowing adequate recovery without exacerbating symptoms. ${ }^{2,4,5}$ Use of the ACE assessment was evaluated in the emergency department setting by Zuckerbraun et al. ${ }^{17}$ In that study, the authors thought that the use of the ACE tool in the emergency department increased patients' mean reported total postconcussion symptom scores and that the self-reported return to normal activity was significantly longer. This result was attributed to enhanced knowledge of concussion symptomatology and improved observance of emergency department discharge instructions. Additionally, the investigators demonstrated increased patient 
follow-up and improved recall of and adherence to emergency department discharge recommendations. In summary, the ACE assessment provides a systematic protocol for mTBI diagnosis and evaluation, and assists in providing appropriate clinical guidance for patient management and referral.

\section{Other Tools: SCAT 3 and ImPACT}

The SCAT3 and Child SCAT3 are standardized tools for evaluating injured, potentially concussed athletes, age 13 years and older and ages 5-12, respectively.,14 Both of these tools are more applicable to sports and patients intending to return to play. There is less systematic guidance for children injured through nonsports-related mechanisms whose goal is to return to school. The ImPACT Concussion Management Model is a 5-step multidisciplinary approach to concussion treatment for athletes. It includes a partnership between health care professionals, athletic trainers, and parents to provide training and resources before, during, and after a concussion occurs. This 30-minute computer-administered test requires a comparative baseline prior to the beginning of the sports season and is typically administered in a separate, quiet room in the clinic. Although baseline studies may be free, fees may be associated with postinjury test administration. ${ }^{8}$

Unlike its predecessors, the ACE assessment is universally applicable to multiple age groups and is not limited to sports-related mechanisms of injury; therefore, it can be used in the context of return to school activities. Moreover, it is free, portable, easily administered, and can be used to recognize patients who experience physical, cognitive, emotional, and sleep difficulties postconcussion. ${ }^{2,4,5}$ In this study, we reported on use of the ACE tool in children age 5 or older, although we have used it in children younger than 5 years. In younger children we have had to rely more on the parental report of symptoms because some of the children did not have the language skills to discuss symptoms. We believe it was beneficial in providing an organized framework for evaluating how the patients were doing postinjury as well.

\section{Limitations and Future Directions}

The findings in this study must be interpreted in the context of its limitations. Previous studies have demonstrated that longitudinal research on pediatric TBI has high rates of attrition. In 1 study, only $32 \%$ of patients followed up with a primary care provider or concussion specialist by Week 4 after their injury, and 39\% reported no followup after their emergency department visit for concussion. ${ }^{13}$ From the original 91 patients with mTBI, 12 children were lost to follow-up. These results are probably due to patients moving away, communication obstacles, transportation difficulties in keeping the appointment, and lack of followup at the family's discretion due to symptom improvement. The latter may have resulted in overreporting the number of patients with problems if one hypothesizes that the patients who were doing well may not have come back for follow-up. Although this study suggests that patients with psychiatric and/or developmental comorbidities and positive CT findings trended toward longer follow-up duration and increased number of symptoms on ACE inventory, the results are limited by the small sample size. Recall bias is a potential limitation because patients were required to remember their symptoms over a period of time. However, by interviewing both the accompanying family members and the patient, we believe that more complete symptom data were obtained, diminishing recall bias.

\section{Conclusions}

We believe that the value of extended follow-up in patients with mTBI is couched in early identification of patients who are having persistent problems. It is recognized that mTBI is not as mild as was once thought for many patients. More than half $(60 \%)$ of patients in our study had persistent mTBI symptoms at the first clinic visit. A systematic evaluation of the patient and the patient's symptomatology assists in early identification of those at risk (who may not have attributed their symptoms to concussive sequelae) and educates the parents or caregivers on what to watch for. It has been our experience that many of these patients have difficulty in school after mTBI, and that schools often do not appropriately identify patients as having issues related to concussion and thus do not offer academic accommodations to these patients without intervention from a health care provider. Primary care providers are often not comfortable managing these issues. We believe that early identification and intervention ultimately results in better outcomes for these patients, and cuts down the number of patient and/or family calls and unscheduled visits to the emergency department for persistent symptoms.

Our data suggest that in patients with mTBI, those with developmental and/or psychiatric comorbidities, or cerebral or calvarial injury trended toward an increased number and prolonged duration of symptoms after injury compared with those without these comorbidities or concurrent injuries. Despite a lack of statistical significance, the results demonstrate a tendency toward a more difficult recovery for patients with psychiatric and/or developmental comorbidities. The results of this study promote the need for appropriate management and referral of pediatric patients with mTBI and comorbid psychiatric diagnoses or concurrent cerebral and calvarial injury. When symptoms persist over time, referral to specialized cognitive rehabilitation and neuropsychological testing is suggested to ensure optimal recovery from symptoms with appropriate professional guidance. Systematic serial ACE assessments are useful in guiding clinicians in providing care and for parental and school counseling. Universally applicable to multiple ages and mechanisms of injury, this evaluation provides a basis for early identification of at-risk patients, enabling more timely referrals for cognitive rehabilitation and neuropsychological testing.

\section{References}

1. Barlow KM, Crawford S, Stevenson A, Sandhu SS, Belanger F, Dewey D: Epidemiology of postconcussion syndrome in pediatric mild traumatic brain injury. Pediatrics 126:e374e381, 2010

2. Centers for Disease Control and Prevention: Heads Up: Facts for Physicians About Mild Traumatic Brain Injury. 
(http://www.concussiontreatment.com/images/CDC_Facts_ for_Physicians_booklet.pdf) [Accessed January 21, 2016]

3. Centers for Disease Control and Prevention: Traumatic Brain Injury in the United States: Fact Sheet. (http://www. cdc.gov/traumaticbraininjury/pdf/BlueBook_factsheet-a.pdf) [Accessed January 21, 2016]

4. Gioia GA: Pediatric assessment and management of concussions. Pediatr Ann 41:198-203, 2012

5. Gioia GA, Collins M, Isquith PK: Improving identification and diagnosis of mild traumatic brain injury with evidence: psychometric support for the acute concussion evaluation. J Head Trauma Rehabil 23:230-242, 2008

6. Goodman JC: Pathologic changes in mild head injury. Semin Neurol 14:19-24, 1994

7. Harmon KG, Drezner JA, Gammons M, Guskiewicz KM, Halstead M, Herring SA, et al: American Medical Society for Sports Medicine position statement: concussion in sport. $\mathbf{B r}$ J Sports Med 47:15-26, 2013

8. Iverson GL, Lovell MR, Collins MW: Immediate postconcussion assessment and cognitive testing (ImPACT). Normative data. Version 2.0 only. ImPACT. (https://www. impacttest.com/pdf/ImPACT_Normative.pdf) [Accessed January 21, 2016]

9. Kelly JP: Traumatic brain injury and concussion in sports. JAMA 282:989-991, 1999

10. McCrory P: Future advances and areas of future focus in the treatment of sport-related concussion. Clin Sports Med 30:201-208, xi-ii, 2011

11. McCrory P: Sports concussion and the risk of chronic neurological impairment. Clin J Sport Med 21:6-12, 2011

12. McCrory P, Meeuwisse WH, Aubry M, Cantu B, Dvorák J, Echemendia RJ, et al: Consensus statement on concussion in sport: the 4th International Conference on Concussion in Sport held in Zurich, November 2012. Br J Sports Med 47:250-258, 2013

13. McCrory P, Meeuwisse W, Johnston K, Dvorak J, Aubry M, Molloy M, et al: Child Sports Concussion Assessment Tool3rd edition. Br J Sports Med 47:263, 2013

14. McCrory P, Meeuwisse W, Johnston K, Dvorak J, Aubry M,
Molloy M, et al: Sports Concussion Assessment Tool-3rd edition. Br J Sports Med 47:259, 2013

15. Neal MT, Wilson JL, Hsu W, Powers AK: Concussions: what a neurosurgeon should know about current scientific evidence and management strategies. Surg Neurol Int 3:16, 2012

16. Zemek RL, Farion KJ, Sampson M, McGahern C: Prognosticators of persistent symptoms following pediatric concussion: a systematic review. JAMA Pediatr 167:259-265, 2013

17. Zuckerbraun NS, Atabaki S, Collins MW, Thomas D, Gioia GA: Use of modified acute concussion evaluation tools in the emergency department. Pediatrics 133:635-642, 2014

\section{Disclosures}

The authors report no conflict of interest concerning the materials or methods used in this study or the findings specified in this paper.

\section{Author Contributions}

Conception and design: all authors. Acquisition of data: all authors. Analysis and interpretation of data: all authors. Drafting the article: all authors. Critically revising the article: all authors. Reviewed submitted version of manuscript: all authors. Approved the final version of the manuscript on behalf of all authors: Ackerman. Statistical analysis: all authors.

\section{Supplemental Information \\ Previous Presentations}

Portions of this study were presented in poster form at the Pediatric Section Meeting, Amelia Island, FL, in December 2014.

\section{Correspondence}

Laurie L. Ackerman, Goodman Campbell Brain and Spine/Indiana University, James Whitcomb Riley Hospital for Children, 705 Riley Hospital Dr., Rm. 1134, Indianapolis, IN 46202-5200. email: lackerma@iupui.edu. 